\title{
Delinquenz kultursensibel erklären - ein theoretisches Rahmenmodell
}

\author{
Stefanie Schmidt ${ }^{1} \cdot$ Tony Ward $^{2}$
}

Eingegangen: 9. Oktober 2020 / Angenommen: 9. November 2020 / Online publiziert: 7. Dezember 2020

(c) Der/die Autor(en) 2020

\section{Zusammenfassung}

Die hohe kulturelle Diversität der Straffälligenpopulation bringt eine ganze Reihe an Herausforderungen für Forschung und Praxis mit sich. Diesen Herausforderungen ist mit gängigen Erklärungsmodellen von Delinquenz jedoch kaum zu begegnen. Einerseits bestehen erhebliche Zweifel an deren allgemeiner Gültigkeit im interkulturellen Kontext, weil sie fast ausschließlich für den euroamerikanischen Raum entwickelt wurden. Andererseits bauen viele der Theorien auf dem Konzept der dynamischen Risikofaktoren auf, welches vage definiert ist. Viele dieser Theorien können Delinquenz kaum erklären, weil sie die zugrunde liegenden psychologischen Mechanismen nur wenig spezifizieren. Mit dem Cultural Agency-Model of Criminal Behavior (CAMCB) schlagen die Autoren ein Rahmenmodell vor, das auf die zugrunde liegenden individuellen psychologischen Mechanismen zielgerichteten Handelns abhebt und dabei systematisch zwischen universellen Komponenten und deren kulturell und individuell gefärbten Ausprägungen differenziert. Im CAMCB wird der Einfluss der kulturellen Sozialisation nicht an eine geografische oder ethnische Herkunft gebunden, sondern an kulturelle „traits“ (z. B. interdependenter Verarbeitungsstil), welche die Verhaltensgenese allgemein beeinflussen. An einem Beispiel wird gezeigt, dass delinquentes Verhalten je nach angenommenem kulturellen Trait unterschiedlich erklärt werden kann, während sich die Situation und die Reaktion nicht unterscheiden müssen. Wesentlich sind: erstens die Identifikation der universellen Verhaltenskomponenten, zweitens die Beschreibung deren kulturell und individuell gefärbter Ausprägungen und drittens die Erklärung von Delinquenz auf der Grundlage dieses Wissens. Dieses stufenweise Vorgehen erscheint nicht nur für die kriminalpsychologische Theorieentwicklung von Bedeutung, sondern auch für die forensische Praxis, bei der für jeden Einzelfall kultursensibel eine individuelle Delinquenztheorie formuliert werden muss.

Schlüsselwörter Dynamische Risikofaktoren · Interdependentes Selbstkonzept · Kriminaltheorien · Straffälligkeit · Agency

Dr. rer. nat. Stefanie Schmidt

st.schmidt@hu-berlin.de

Prof. Dr. Tony Ward

Tony.Ward@vuw.ac.nz

1 Humboldt-Universität zu Berlin, Rudower Chaussee 18, 12489 Berlin, Deutschland

2 School of Psychology, Victoria University of Wellington, PO Box 600, Wellington, Neuseeland 


\title{
Explaining delinquency in a culture-sensitive manner-a theoretical framework
}

\begin{abstract}
Because offender populations are highly diverse research and practice face many challenges; however, meeting these challenges with common theories of crime is hardly possible. On the one hand, there is reasonable doubt about their cultural equivalence as these theories were mainly formulated for a Euro-American cultural context. On the other hand, most of the theories are based on the concept of dynamic risk factors, which is vaguely defined. Moreover, most theories do not explain criminal behavior as they do not specify the underlying psychological mechanisms. We propose a framework the Cultural Agency-Model of Criminal Behavior (CAMCB), which depicts the general processes underpinning human beings' goal-directed behavior. The CAMCB differentiates between universal components of behavior and the way cultural traits shape the content and operation of these components. The influence of cultural socialization on psychological processes is explained without referring to a person's ethnic background or heritage but by referring to cultural traits (e.g. interdependency), which shape the agency processes in general. By means of example we show that criminal behavior can be explained differently depending on the cultural trait even though the situation and reaction might be the same. It is essential to first identify universal components of a behavioral process, second, describe how cultural traits influence this process and third, explain criminal behavior on the basis of this knowledge. This stepwise approach is not only central for theory development in criminal psychology but also for forensic practice, as practitioners have to formulate an individual theory of crime for every single person in a culture-sensitive manner.
\end{abstract}

Keywords Dynamic risk factors $\cdot$ Interdependent self-construal $\cdot$ Theory of crime $\cdot$ Offending $\cdot$ Agency

Die hohe Diversität der Straffälligenpopulation (Aebi et al. 2015) stellt Wissenschaftler und Praktiker vor enorme Herausforderungen. Damit es zu keiner systematischen Benachteiligung einzelner Gruppen von Straffälligen kommt, sollten alle Anstrengungen unternommen werden, um die Risikobeurteilungen und Rehabilitationsmaßnahmen für alle gleichermaßen valide und wirksam zu gestalten (Jones et al. 2002; Hart 2016; Shepherd und Lewis-Fernandez 2016). Spätestens seit Anfang der 2000er-Jahre entwickelte sich eine lebhafte wissenschaftliche Debatte um die Güte gängiger Prognosemethoden. Damals klagte der kanadische Inhaftierte Jeffrey G. Ewert, der sich einer Gruppe der Aborigines zugehörig fühlt, immer wieder gegen das kanadische Justizsystem, weil er die Anwendung von bestimmten aktuarischen Kriminalprognoseinstrumenten bei seiner kulturellen Gruppe für unzulänglich hielt, was weitrechende Konsequenzen hatte (Hart 2016). Wie sich diese Debatte in den empirischen Befunden widerspiegelt, lässt sich gut an den sehr breit beforschten statistisch-nomothetischen Instrumenten rund um die „Central-Eight“-Risikofaktoren verdeutlichen (z. B. Level of Service Inventory-Revised [LSI-R]; Andrews und Bonta 1995). In Metaanalysen zu den LS-Instrumenten zeigte sich, dass ethnische Minderheiten auf fast allen Central-Eight-Risikofaktoren höhere Werte hatten als die Vergleichsgruppe (Olver et al. 2014). Darüber hinaus fand sich, dass die prädiktive Validität bei ethnischen Minderheiten etwas geringer ausfiel und die Validität über die verschiedenen Stichproben hinweg stärkeren Schwankungen unterworfen war als bei der Vergleichsgruppe (Wilson und Gutierrez 2014; Olver et al. 2014). Ob diese Unterschiede jedoch mit kulturellen Variablen zu tun ha- ben oder nur Artefakte sind, kann bisher kaum aufgeklärt werden. Es ist auch nicht möglich zu erklären, warum je nach kulturellem Kontext bestimmte dynamische Risikofaktoren mehr oder weniger mit Delinquenz assoziiert sind. Diese Unzulänglichkeit hängt maßgeblich mit den Schwächen des gemessenen Konzeptes dynamischer Risikofaktoren (DRF) zusammen (Schmidt et al. 2020). In dem vorliegenden Beitrag analysieren die Autoren zunächst diese Schwächen. Darauffolgend stellen sie eine alternative theoriegeleitete Herangehensweise zum Verständnis kultureller Unterschiede bei der Aktualgenese delinquenten Verhaltens - das „Cultural Agency-Model of Criminal Behavior“" (CAMCB) - vor und illustrieren die Anwendung an einem vergleichenden Beispiel. Neben der Ableitung praktischer Implikationen besprechen die Autoren das Modell schließlich entlang gängiger Gütekriterien von Theorien und geben einen Ausblick zu möglichen Weiterentwicklungen und Validierungsstudien.

\section{Konzeptionelle Schwächen gängiger Ansätze aus theoretischer Perspektive}

Das LSI-R ist ein statistisch-nomothetisches Instrument zur Kriminalprognose, welches nicht nur statische Risikofaktoren erfasst, die sich in Rückfallstudien als valide Prädiktoren gezeigt haben. Durch die zusätzliche Erhebung von DRF fußt das Verfahren auf theoretischen Überlegungen zur Entstehung und zur Aufrechterhaltung von Delinquenz. Andrews und Bonta (2010) nehmen an, dass die DRF in irgendeiner Form ursächlich für die Delinquenz sind. Nach 
der Theorie des General Personality and Cognitive Social Learning (GPCSL) wirken die Central Eight zusammen mit einer Situation auf die Entscheidungsfindung, welche einer delinquenten Handlung vorausgeht (Bonta und Andrews 2017). Wenn sich diese Faktoren nun (z. B. durch Interventionen) verändern, dann reduziert sich auch das Rückfallrisiko, so die Annahme. Somit sollte sich die Behandlungsplanung an diesen DRF oder ,criminogenic needs“ ausrichten (Andrews und Bonta 2010). Dieser Ansatz hat die forensische Forschung und Praxis enorm bereichert und vorangebracht. Andrews und Bonta gelang es, das Bemühen um Rehabilitation gegenüber rein punitiven und kontrollierenden Herangehensweisen zu stärken und die Behandlungsplanungen zu systematisieren (Schmidt 2019). Trotz dieser Gewinne hat die zugrunde liegende Theorie bzw. das Konstrukt der DRF als solches einige Nachteile.

Erstens handelt es sich bei den DRF (auch in der zugrunde gelegten Theorie) um sehr vage definierte, heterogene Konstrukte (Ward 2016). Unter den einzelnen Risikofaktoren (z.B. antisoziales Persönlichkeitsmuster) werden sehr viele unterschiedliche Dinge (z. B. Impulsivität, abenteuerliches Stimulationsbedürfnis, generalisierte Probleme, Aggression, Gefühlskälte, Empathiemangel) aus vielen verschiedenen konzeptuellen Kategorien (z. B. Emotionen und psychische Zustände, Verhaltensweisen, soziale Faktoren) zusammengefasst (Ward und Fortune 2016). Diese einzelnen Aspekte können jeder für sich unterschiedlich auf delinquentes Verhalten wirken, was es schwer macht, den genauen Einfluss des Faktors zu erklären. In diesem Sinne ist auch unklar, wie die Indikatoren dieser DRF mit ihrem Konstrukt oder auch untereinander genau zusammenhängen (Via et al. 2016).

Zweitens sind die Konstrukte sehr unspezifisch formuliert, was zu ähnlichen Problemen bei der Erklärung von Delinquenz führt (Ward und Fortune 2016). So mag es sich z.B. bei dem Faktor antisoziales Persönlichkeitsmuster einerseits um ein Symptom (z. B. aggressives Verhalten), andererseits um einen kontextuellen Einfluss (z.B. parasitärer Lebensstil) oder aber um eine tatsächliche Ursache für eine delinquenzbegünstigende Handlungsentscheidung (z.B. mangelnde Fähigkeit zum Bedürfnisaufschub) handeln (Schmidt et al. 2020).

Drittens sind die Definitionen der DRF untrennbar mit sozialen Normen verbunden. Sie beziehen sich direkt auf bestimmte Verhaltensweisen, die durch eine Gesellschaft als schädlich bewertet wurden (Ward 2016). Durch die Label „kriminell“, „kriminogen“, ,antisozial“ oder „problematisch" werden unerwünschte Zustände auf Basis geteilter sozialer Werte und Normen beschrieben (Cooper 2013). Weil sich Werte und Normen zwischen Kulturen unterscheiden, können diese Faktoren nicht ohne Weiteres auf andere soziale oder kulturelle Kontexte übertragen werden. Denn ,risk item content often reflects the practices, percep- tions, norms, belief systems, and behavioral expectations of Western culture" (Shepherd und Lewis-Fernandez 2016, S. 429). Damit erfolgt eine normative Festlegung auf eine ganz bestimmte Vorstellung über die Welt und menschliches Zusammenleben.

Viertens handelt es sich bei den Theorien, die DRF als Erklärungen verwenden, um funktionale Theorien; sie beschreiben keine kausalen Wirkmechanismen. Die Annahme, dass eine therapeutisch initiierte Reduktion der DRF das Risiko einer erneuten Straftat senkt, impliziert jedoch, dass es sich bei den Faktoren um Ursachen für Delinquenz handelt (Heffernan et al. 2019). Mit der Einführung der DRF sind diese also nicht bloß Merkmale, die mit Rückfälligkeit in Verbindung stehen, sondern sie sollen die Delinquenz erklären (Fortune und Heffernan 2018). Um als kausale Erklärung zu fungieren, müssen diese Faktoren aber nicht nur statistisch relevant sein und gut begründet aneinandergereiht werden. Wesentlich ist, dass die kausalen Zusammenhänge mit Delinquenz möglichst genau beschrieben werden (Heffernan und Ward 2017). Die vage Definition dieser Konstrukte macht eine solch kausale Referenz aber schwer, weil unklar ist, welche Aspekte der heterogenen Konstrukte auf welche Weise mit Delinquenz zusammenhängen. Dies zeigt sich empirisch auch in den methodischen Schwierigkeiten sowie uneindeutigen Befunden zur rückfallprädiktiven Wirkung von Veränderungen dieser Faktoren (Heffernan et al. 2019). Auch wenn Andrews und Bonta die kausalen Wirkmechanismen post hoc mittels einer Vielzahl anderer Theorien zu erklären suchen, geschieht dies eher unstrukturiert und vage (Fortune und Heffernan 2018). Der Leser weiß nicht, nach welcher Theorie welche spezifischen Aspekte welches DRF wie genau mit Delinquenz zusammenhängen.

Wenn DRF Delinquenz also schon innerhalb der Population, in der die Theorie entwickelt wurde, nicht kohärent erklären können, ist das für diverse kulturelle Kontext erst recht kaum möglich. Zu erklären, auf welche Weise ein Risikofaktor in anderen kulturellen Kontexten wirkt, ist schwer, weil man dafür auf die ursächlichen Wirkmechanismen Bezug nehmen müsste, die schon in der Originaltheorie kaum spezifiziert sind (Schmidt et al. 2020).

Diese Argumentation orientiert sich beispielhaft an der Theorie von Andrews und Bonta, weil das Konzept der DRF sehr stark von den beiden geprägt wurde (Gottfredson und Moriarty 2006). Die aufgezeigten Schwächen finden sich aber auch in anderen theoretischen Ansätzen. Auch Erklärungsansätze delinquenter Entwicklungsverläufe (z. B. biopsychosoziales Modell kumulativer Risikofaktoren, Lösel und Bliesener 2003; Developmental Pathways in Juvenile Externalizing and Internalizing Problems, Loeber und Burke 2011; Integrated Cognitive-Antisocial Potential, Farrington 2020), die in der Kriminalpsychologie verbreitet sind, bauen mehr oder minder auf dem Konzept der DRF auf und 
spezifizieren die zugrunde liegenden psychologischen Mechanismen kaum (Dahle et al. 2020). Es handelt sich damit um ein übergreifendes Problem in der kriminalpsychologischen Theoriebildung, welches weitreichende Konsequenzen für die forensische Praxis hat (Ward 2020).

\section{Hindernisse in der forensischen Praxis}

In der forensischen Praxis nimmt die Anwendung von Theorien zur Erklärung von Delinquenz einen hohen Stellenwert ein. Bei der idiografisch geleiteten Prognosemethode ist nach Dahle (2010) ein systematischer Bezug zu etablierten (kriminalpsychologischen) Theorien bei der Formulierung der individuellen Kriminaltheorie unabdingbar. So sollte auch die individuelle Relevanz der Risikofaktoren, die z.B. mittels LSI-R identifiziert wurden, für den Einzelfall bewertet und der Zusammenhang im Rahmen der Deliktdynamik beschrieben werden. Auf diese individuellen Kriminaltheorien bauen wiederum die Behandlungsansätze auf. Um sicherzustellen, dass die Behandlung auch die Rückfallwahrscheinlichkeit reduziert, sollte genau beschrieben werden, welche Veränderungen welcher ursächlichen Faktoren durch welche Intervention angestrebt werden und welchen Einfluss dies auf die Deliktdynamik und in der Folge auf die Rückfallwahrscheinlichkeit hat (Heffernan et al. 2019).

Weil für die Theoriebildung im Einzelfall also auf etablierte Theorien aus der Fachliteratur zurückgegriffen wird, wirken sich die aufgezeigten Schwächen der DRF in der Theoriebildung auch auf die forensische Praxis aus (Ward 2020). Wenn die Definitionen vage und die zugrunde liegenden Mechanismen kaum spezifiziert sind, muss der Praktiker dies nach Gutdünken übernehmen. Diese Herausforderung wird durch die Heterogenität der Straffälligenpopulation noch größer, weil sich dadurch zusätzlich ständig variierende Ausprägungen der unterschiedlichen Facetten einzelner Risikofaktoren ergeben (Schmidt 2018; Schmidt et al. 2018). Diese Unterschiede gilt es, in der Urteilsbildung theoretisch einzuordnen und auch im Vergleich zu bewerten. Die theoriegeleitete Einordnung sollte deshalb schwerfallen, weil ein solcher Bezug zur Theorie nur vage vorhanden ist (Schmidt et al. 2020). Auf welche konkreten Mechanismen hat z. B. die Familie Einfluss bei der individuellen Entscheidung, delinquent zu handeln? Wie ist vor diesem Hintergrund die starke Einbindung in den familiären Kontext bei Personen aus einem kollektivistisch geprägten Kulturkreis zu bewerten? Aber auch die vergleichende Bewertung erscheint mit Blick auf die dünne und heterogene empirische Befundlage (Hart 2016; Shepherd und Lewis-Fernandez 2016) problematisch. Ist z. B. der Risikofaktor Familie bei Personen aus einem kollektivistisch geprägten Kulturkreis im Durchschnitt geringer ausgeprägt als bei Personen aus einem individualistisch geprägten Kulturkreis? Wenn ja, warum? Und wirkt sich dies auf die Validität dieses Prädiktors aus? Es fehlen demnach sowohl theoretisch als auch empirisch Referenzrahmen, die eine solche Urteilsbildung transparent gestalten würden. Infolge dieses Mangels kann die Praxis nur auf zwei Strategien ausweichen: 1) Die nötige Anpassung bleibt aus, weil keine theoretisch fundierte Einordnung bzw. kein Vergleich stattfindet, d.h. alle Personen werden ungeachtet ihrer Werte und Hintergründe gleich bewertet, was Ethnozentrismus und eine wenig valide Beurteilung zur Folge haben kann. 2) Die nötige Anpassung erfolgt nach intuitiver Vorgehensweise, was zu Bias aufgrund von Stereotypen und anderen Urteilsfehlern und damit gleichfalls zu einer wenig validen Beurteilung führen kann.

Zusammenfassend lässt sich festhalten, dass DRF zur Erklärung von Delinquenz wenig geeignet sind (Ward 2016). Dies behindert nicht nur die Theoriebildung und Theorieentwicklung, die auf DRF aufbauen. Auch in der Praxis lassen sich mit diesen Faktoren die Delinquenz des Einzelfalls kaum erklären bzw. darauf aufbauend konkrete Interventionsmaßnahmen ableiten (Ward 2020). Im interkulturellen Kontext kommen diese Schwächen, insbesondere die festgeschriebene Bindung an bestimmte Werte und Normen, noch stärker zum Tragen. Dabei ist Kultur nicht allein mit Herkunft oder einer spezifischen Sozialisationsform gleichzusetzten. Kultur ist die geteilte Lebensweise einer Gruppe von Menschen (Berry et al. 2011). Damit bewegt sich die Erklärung delinquenten Verhaltens (zumindest in der Praxis) meistens in einem interkulturellen Kontext.

Auch viele andere Erklärungsmodelle basieren auf einer Auflistung von DRF oder teilen die Grundprobleme dieses Konzeptes. Um die Hindernisse in der (kriminal-)psychologischen Theoriebildung zu umgehen (Ward 2020) und Alternativen auch für die Praxis bereitzustellen, braucht es aus Sicht der Autoren eine theoretische Grundlage, die die Ursachen und Wirkmechanismen delinquenten Verhaltens beschreibt, bei der kulturbedingte Spezifika systematisch und transparent einbezogen werden können und die gleichzeitig auf verschiedene kulturelle Kontexte anwendbar ist. Im Folgenden beschreiben wir ein mögliches Rahmenmodell, welches bereits ausführlich in einer englischsprachigen Veröffentlichung vorgestellt wurde (Schmidt et al. im Druck). Dieses Modell strukturiert den Erklärungsprozess, beschreibt basale und universelle psychologische Prozesse und formuliert Kriterien für die Bewertung von Verhalten. Es handelt sich nicht um eine spezifische Theorie, sondern um ein Modell zur Theoriebildung, wie spezifische kultursensible Theorien für einzelne Gruppen oder Einzelfälle formuliert werden können. 


\section{The Cultural Agency-Model of Criminal Behavior}

\section{Grundprinzipien}

Wir sehen delinquentes Verhalten als eine Form von Verhalten an, das auf die gleiche Weise erklärt werden kann wie jedes andere Verhalten auch. Weil dieses (delinquente) Verhalten hochkomplex ist und zahlreiche Einflussfaktoren auf unterschiedlichsten Erklärungsebenen dieses Verhalten konstruieren (Thagard 2019), halten wir zuvorderst fest, dass die individuell-psychologische Erklärungsebene nur eine von vielen, ineinander verschachtelten Erklärungsebenen ist. Die individuellen Fähigkeiten und verfügbaren Informationen (z.B. die individuellen Einstellungen) werden durch die Interaktion mit anderen (z.B. Freunde und Bekannte) geformt und gestalten diese gleichfalls mit. Interaktionen finden wiederum in bestimmten Institutionen (z. B. Schule) mit spezifischen Rollenerwartungen und Normen statt, welche selbst durch den rechtlichen und ökonomischen Kontext gebildet werden, der wiederum bestimmten Umweltbedingungen und historischen Entwicklungen unterliegt. Diese verschiedenen Ebenen sind durchlässig, bedingen sich gegenseitig und sind alle relevant für die Erklärung von (delinquentem) Verhalten (Strauss-Hughes et al. 2019). Nicht nur Verhalten als solches kann über diese Erklärungsebenen beschrieben werden, sondern auch das, was Kultur ausmacht. Dabei entwickelt sich Kultur gewissermaßen fortlaufend dynamisch über all diese Ebenen und deren Austausch hinweg (Markus und Kitayama 2010).

Das CAMCB ist so konstruiert, dass es pluralistische Erklärungen ermöglicht. Es fokussiert im engeren Sinne aber auf die individuell-psychologische Ebene im Querschnitt. Dieser individuell-psychologischen Perspektive liegt die Annahme zugrunde, dass jedes Verhalten Ausdruck von Agency, also der Fähigkeit, zielgerichtet zu handeln, ist. Dafür werden die Zukunft simuliert, eine Intention gebildet,
Handlungspläne umgesetzt und die Ergebnisse schließlich evaluiert (Bandura 2006). Diese Annahme beruht auch auf Theorien und empirischer Evidenz, dass jedes Leben auf die physische (und bei Menschen auch die soziale) Umgebung reagiert, um erwünschte Zustände zu erreichen und schädliche zu vermeiden (Maiese 2015). Wenngleich Agency als grundlegender Prozess für Menschen universell sein dürfte, so scheint das Erleben davon, was Agency ausmacht und wie sich der Agens konstituiert, in unterschiedlichen kulturellen Kontexten zu variieren. Erlebt sich eine Person beispielsweise als autonomes Selbst, ist die Handlung eher Ausdruck von Persönlichkeit und Selbstbestimmtheit, während die Handlung einer Person, die sich als Teil eines Systems wahrnimmt, eher Ausdruck von Rollenerwartungen und den Bedürfnissen anderer ist (Markus 2016; Markus und Kitayama 2010).

Das Anliegen ist es, dieser Flexibilität in der Erklärung Raum zu geben und die verschiedenen Einflüsse transparent zu machen. Deshalb schlagen die Autoren eine schrittweise und aufeinander aufbauende Herangehensweise bei der Erklärung von (delinquentem) Verhalten vor (Abb. 1):

1. Nehmen die Autoren an, dass es bestimmte Komponenten und Mechanismen im Agency-Prozess gibt, die immer relevant sind. Beispielsweise strebt jeder Mensch nach der Erfüllung subjektiv bedeutsamer Bedürfnisse, die seine Handlungsplanung und sein Verhalten leiten (Ward und Stewart 2003). Das CAMCB stützt sich in der Beschreibung dieser Komponenten auf grundlagenpsychologische Konzepte menschlichen Erlebens und Verhaltens, die so allgemein sind, dass sie als kulturell universell gelten dürften. Universell ist in diesem Sinne die grundlegende biologische Befähigung zum Handeln, die Individuen aller Kulturen vereint, wie beispielsweise die Fähigkeit zu einem Selbstkonzept oder der Gebrauch von Selbstregulationsmechanismen (Del Giudice 2018).

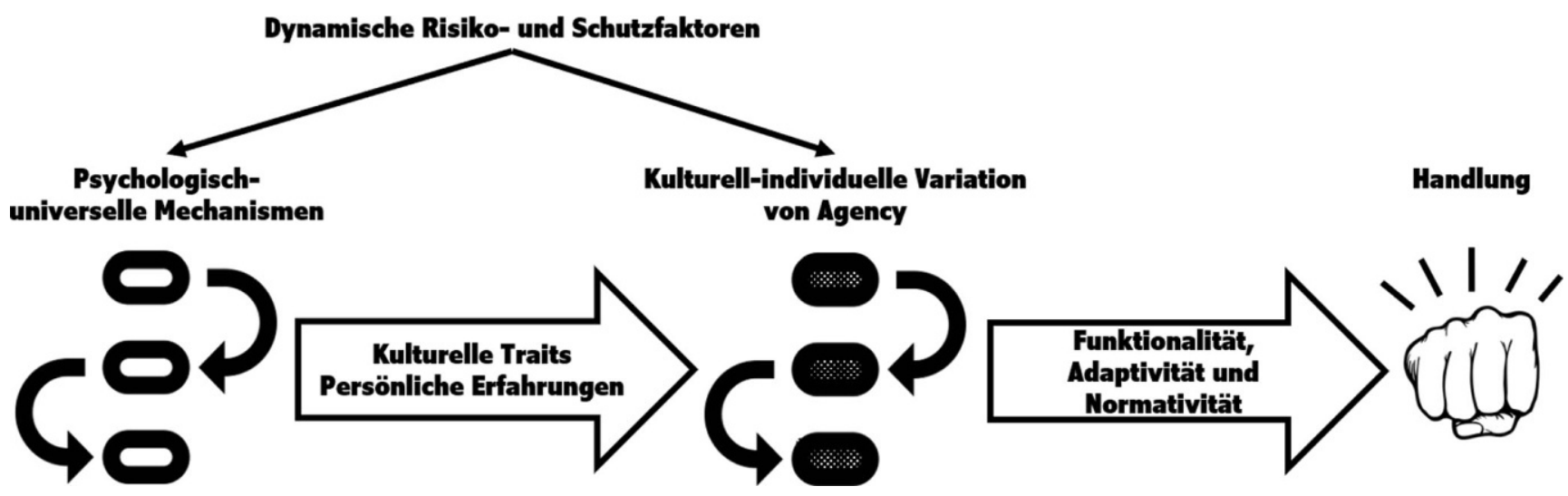

Abb. 1 Psychologisch-universelle Mechanismen, die kulturell-individuelle Variation von Agency und das Ergebnis als eine schrittweise aufeinander aufbauende Erklärung von delinquentem Verhalten 
2. Dieses Gerüst der Handlungskomponenten wird durch wechselseitige Anpassungsprozesse zwischen Umwelt und Individuum (kulturelle Nischenkonstruktion) geformt (Sterelny 2018). Diese kulturbedingten Ausprägungen nennen wir im Folgenden kulturelle Traits. Sie funktionieren wie Linsen, durch die sich Verhalten verstehen lässt, weil sie den Agency-Prozess und dessen Komponenten grundlegend beeinflussen (Markus und Kitayama 1991). Es sind aber nicht nur Erfahrungen aus kulturellen Lernprozessen, welche die Inhalte und Prozesse dieser Handlungskomponenten prägen. Auch ganz singuläre, individuelle Erfahrungen determinieren Handlungsentscheidungen (Matsumoto 2007).

3. Erst unter der Annahme einer spezifischen kulturell und individuell geprägten Ausformung von Agency erfolgt die Bewertung der Handlung und der ihr zugrunde liegenden Komponenten und Prozesse. Diese Bewertung bezieht sich auf die Funktionalität, die soziale Adaptivität und die Legalität. Demnach kann eine Handlung (z. B. Totschlag) gemäß der hierzulande geltenden Rechtsnorm als illegal bewertet werden. Ursache für diesen Normverstoß kann etwa eine Dysfunktionalität in einzelnen Komponenten des Agency-Prozesses sein. Beispielsweise kann eine Läsion im orbitofrontalen Kortex eine mangelnde Fähigkeit zur Empathie verursacht haben, was die Handlung (mit)erklären kann (Shamay-Tsoory et al. 2010). Es wäre aber auch möglich, dass der Agency-Prozess vollkommen funktional ist und die Ursache für den Normverstoß in der Maladaptivität (einzelner Komponenten) des Agency-Prozesses liegt. So könnten z.B. die individuell angewendeten Strategien zur Emotionskontrolle und zur Konfliktlösung insofern maladaptiv sein, als dass die eigenen Interessen nicht auf prosoziale Weise durchgesetzt werden können. Der Normverstoß ist aber auch bei unbeeinträchtigter Funktionsweise und bei einer aus Sicht des Individuums hohen Adaptivität erklärbar. So könnte ein Totschlag infolge einer als erheblich empfundenen Verletzung der Familienehre, die durch die Tat wiederhergestellt werden kann, als normales Verhalten erlebt werden. Adaptivität ist damit sehr vom Kontext abhängig.

Eine Theorie, welche für einen spezifischen kulturellen Kontext delinquentes Verhalten aus der individuellen Perspektive zu erklären sucht, oder eine für den Einzelfall formulierte individuelle Kriminaltheorie ist unseres Erachtens dann kultursensibel, wenn sie die oben genannten kulturell bedingten Einflüsse explizit berücksichtigt. Sowohl die konkrete Manifestation der einzelnen Komponenten und Wirkmechanismen im Agency-Prozess als auch die Bewertung der sozialen Adaptivität und Legalität des Verhaltens ist von der kulturellen Sozialisation sowie den kulturellen Normen abhängig.

\section{Komponenten und ihre Wirkbeziehungen}

Doch welche Komponenten einer Handlung könnten universell als bedeutsam erachtet werden? Eine Handlung entsteht auf der Grundlage biologischer Befähigungen und zugänglicher Informationen, die verarbeitet werden (Anderson und Bushman 2002; Crick und Dodge 1994; Heffernan und Ward 2017; Sterelny 2018). Wenngleich die biologischen Determinanten sehr bedeutsam sind und für eine umfassende Erklärung nicht vernachlässigt werden sollten, konzentrieren die Autoren sich bei der Beschreibung des CAMCB auf die Informationen, die dem Individuum zugänglich sind. Das CAMCB schlägt eine Möglichkeit vor, wie der Prozess von Agency (Bandura 2006) in seinen Grundzügen beschrieben werden kann. Dabei bedienen wir uns grundlagenpsychologischer Konstrukte und Modelle, die zusammenhängend v. a. von Theorien der sozialen Informationsverarbeitung beschrieben wurden (Wyer 2012). Das CAMCB baut dabei direkt auf das „Reformulated Social Information-Processing Model“ von Crick und Dodge (1994) und dessen Adaptation „Integrated Model of Emotion Processes and Cognition in Social Information Processing" (Lemerise und Arsenio 2000) auf. Diese Modelle liefern eine sequenzielle Beschreibung psychologischer Prozesse, die aggressivem Verhalten zugrunde liegen, und können als empirisch gut validiert angesehen werden (z.B., Horsley et al. 2010; Raaijmakers et al. 2008; Rohlf et al. 2018; Verhoef et al. 2019). Auch das „Predictive Agency Model“" von Heffernan und Ward (2017) wurde für die Konstruktion des CAMCB herangezogen, weil es die Bedeutung von Agency betont und die Ausformung von Agency im Kontext von straffälligem Verhalten kohärent darstellt.

Wie die Abb. 2 verdeutlicht, unterscheiden wir im CAMCB zwischen a) externalen Inputs und Outputs psychologischer Prozesse, b) den sequenziellen Schritten dieser Prozesse-nämlich: 1) Wahrnehmen; 2) Interpretieren; 3) Planen; 4) Handeln; 5) Reflektieren - und c) internalen Determinanten dieser psychologischen Prozesse (mentale Eindrücke und Emotionen).

Mentale Eindrücke sind Wissensnetzwerke oder kognitive Strukturen, die durch Erfahrung konstruiert werden. Sie unterscheiden sich dabei in ihrer Komplexität, Spezifität und den Verbindungen. So können vergangene Erlebnisse miteinander verbunden werden und abstraktere Eindrücke, wie Schemata, Skripte und mentale Modelle des Selbst, der Welt und sozialer Beziehungen, formen (Anderson und Bushman 2002; Agnew 1992; Crick und Dodge 1994; Heffernan und Ward 2017; Wyer 2012). Die Fähigkeit dazu, Eindrücke zu speichern und zu abstrakterem Wissen und Fertigkeiten zu formen, ist universell. Der Inhalt dieser mentalen Eindrücke ist jedoch sehr variabel und wird entweder mit einer großen Gruppe anderer geteilt, was die Autoren kulturelle Traits nennen, oder er ist sehr individu- 


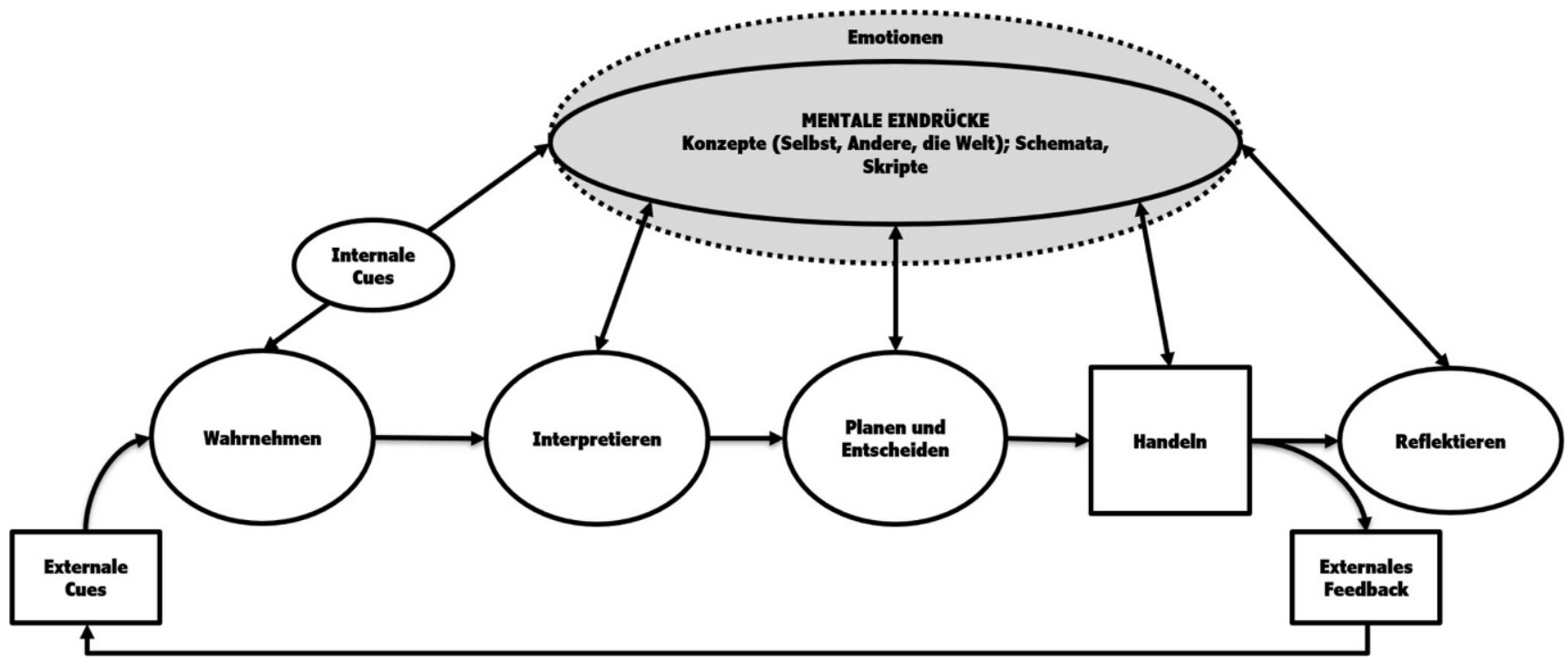

Abb. 2 Die Verhaltensgenese im Cultural Agency-Model of Criminal Behavior (CAMCB); internale Determinanten, die die einzelnen Sequenzen der Verhaltensgenese beeinflussen, sind grau dargestellt; zweiseitige Pfeile zeigen reziproke Verbindungen an; sichtbare Komponenten sind eckig dargestellt, latente und nichtbeobachtbare Komponenten rund

ell. Kulturelle Traits sind also mentale Eindrücke (Chiu und Hong 2007), die gleichfalls eher abstrakt (z. B. Kollektivismus) oder sehr spezifisch (z. B. Riten und Rituale; Oyserman und Yan 2018) sein können. Sie sind hoch funktionale Adaptationen an soziale und physische Umgebungen (Kim und Lawrie 2018), die über soziale Lernprozesse vermittelt wurden, damit gewünschte Ziele erreicht und unerwünschte vermieden werden können (Mesquita et al. 2016). Auf diese Weise beeinflussen kulturelle Traits den gesamten Agency-Prozess, indem sie dem in Abb. 2 dargestellten Modell ähnlich einer Linse die Grundfarbe verleihen. Je nach aktiviertem kulturellen Trait dominieren bestimmte (als normativ erlebte) Emotionen, werden bestimmte cues wahrgenommen, bestimmte Arten von Zielen bevorzugt und ein bestimmtes Handlungsregulationssystem stärker angesprochen.

Dabei wird jeder Schritt im Agency-Prozess einerseits von den mentalen Eindrücken beeinflusst; andererseits produziert bzw. verändert jeder Schritt mentale Eindrücke in einer rekursiven Schleife (Anderson und Bushman 2002; Crick und Dodge 1994). Das bedeutet aber nicht, dass diese Prozesse immer linear ablaufen müssen. Vielmehr scheinen Menschen andauernd wahrzunehmen, zu interpretieren und $\mathrm{zu}$ planen und somit unterschiedliche mentale Eindrücke parallel zu verarbeiten, weshalb ein Schritt im Agency-Prozess nicht unbedingt durch den vorherigen determiniert sein muss (Crick und Dodge 1994). Dennoch lässt sich annehmen, dass die Stärke der mentalen Eindrücke dann am größten ist, wenn sie alle Schritte des Agency-Prozesses kohärent beeinflussen. Die Person interpretiert die Situation und antizipiert potenzielle Konsequenzen, bildet eine Intention, die auf diesem Wissen aufbaut, führt die Handlung wie intendiert aus und ist in der Reflexion mit der Handlung zufrieden. Dieselben mentalen Eindrücke beeinflussen jeden Schritt des Agency-Prozesses und werden durch das Feedback zusätzlich verstärkt (Schmidt et al. im Druck).

Wenngleich die grundlegende Annahme ist, dass jede Handlung, auch eine strafrechtsbewehrte, zielgerichtet ist (Ward 2017), schließt dies nicht aus, dass sehr viele dieser Intentionen und Prozesse unbewusst sind. Doch auch automatisierte oder unbewusste Intentionen waren zu einem Zeitpunkt neu und wurden bewusst verarbeitet und beeinflussen somit Verhalten in der gleichen Weise (Eitam und Higgins 2010), weshalb diese Darstellung für die Erklärung von Verhalten auch in diesen Fällen geeignet erscheint. Weiterführende Erläuterungen zu den einzelnen Komponenten sowie potenzielle Ausformungen dieser Komponenten in unterschiedlichen kulturellen Kontexten finden sich in der englischsprachigen Publikation (Schmidt et al. im Druck).

\section{Beispiel für eine kultursensible Erklärung}

Um die Anwendung des CAMCB zu illustrieren, haben die Autoren eine ambivalente Situation ausgewählt, die eine aggressive Handlung zur Folge haben kann. Zur Verdeutlichung des Kontrastes zweier unterschiedlicher kultureller Traits wird im Folgenden die gleiche Situation einmal aus den Augen einer Person mit einem unabhängigen Verarbeitungsstil und einmal aus den Augen einer Person mit einem interdependenten Verarbeitungsstil betrachtet. Nach Markus und Kitayama $(2010,1991,1998)$ impliziert ein $u n$ abhängiger Verarbeitungsstil eine Selbstwahrnehmung als abgetrennt von anderen und einzigartig aufgrund der individuellen Kombination von internalen Eigenschaften. Dieses 
kulturelle Trait steht in engem Zusammenhang mit übergreifenden Motiven nach Selbstverwirklichung und dem Wunsch, die eigene Individualität auszudrücken. Damit assoziiert sind die Werte Autonomie, Macht und selbsterbrachte Leistung. Im Gegensatz dazu impliziert ein interdependenter Verarbeitungsstil die Wahrnehmung des Selbst als untrennbar mit anderen verbunden, die zu dem bedeutsamen sozialen Kontext gehören. Hier werden v. a. die Motive Anpassung und Norm- bzw. Rollenerfüllung als integraler
Bestandteil des sozialen Miteinanders angesprochen. Dies ist assoziiert mit den Werten Unterordnung, Zurückhaltung, gemeinschaftliche Leistung, Geduld und Fürsorge (ebd.).

In dem folgenden Beispiel betrachten wir diese Situation: Ein junger Mann besucht zusammen mit Freunden eine Bar. Nach einer kurzen Unterhaltung entfernt sich der junge Mann etwas von den Freunden, um an der Theke zu bestellen. Dort wird er von einem weiteren Gast etwas zur Seite geschoben, als dieser an die Bar drängt und seine ei-

Tab. 1 Der Agency-Prozess für einen unabhängigen vs. einen interdependenten Verarbeitungsstil, illustriert am Beispiel einer aggressiven Verhaltensgenese

\begin{tabular}{|c|c|c|}
\hline Komponente & Unabhängiger Verarbeitungsstil & Interdependenter Verarbeitungsstil \\
\hline \multirow[t]{2}{*}{$\begin{array}{l}\text { Mentale } \\
\text { Eindrücke }^{\mathrm{a}}\end{array}$} & $\begin{array}{l}\text { Erleben des Selbst als getrennt und Fokus auf die ein- } \\
\text { zigartigen Charakteristika der Person } \rightarrow \text { Selbstbild, } \\
\text { persönliche Einstellungen und individuelle Bedürfnisse } \\
\text { stärker aktiviert }\end{array}$ & $\begin{array}{l}\text { Erleben des Selbst als untrennbar mit anderen verbunden und } \\
\text { Fokus auf die Gemeinschaft sowie deren Rollenerwartungen } \rightarrow \\
\text { vorgestelltes Fremdbild, soziale Normen und Bedürfnisse anderer } \\
\text { stärker aktiviert }\end{array}$ \\
\hline & Ich bin besser als andere und ihnen überlegen. & Ich bin Teil einer Gruppe, die anderen deutlich überlegen ist. \\
\hline \multirow[t]{2}{*}{ Emotionen $^{\mathrm{b}}$} & $\begin{array}{l}\text { Starkes Arousal bei hoher Autonomie } \rightarrow \text { Begeisterung } \\
\text { und Stolz als präferierte emotionale Zustände; Lange- } \\
\text { weile und Gekränktheit als zu vermeidende Zustände }\end{array}$ & $\begin{array}{l}\text { Geringes Arousal bei hoher Verbundenheit } \rightarrow \text { Genügsamkeit und } \\
\text { Freundlichkeit als präferierte emotionale Zustände; Entrüstung } \\
\text { und Streitlust als zu vermeidende Zustände }\end{array}$ \\
\hline & $\begin{array}{l}\text { Nach einem Misserfolg fühle ich mich selbstunsicher, } \\
\text { was mich ärgert. }\end{array}$ & $\begin{array}{l}\text { Ich möchte dazugehören und habe Angst, der Gruppe zu schaden } \\
\text { oder deren Erwartungen nicht zu erfüllen. }\end{array}$ \\
\hline \multirow[t]{2}{*}{ Wahrnehmen $^{\mathrm{c}}$} & $\begin{array}{l}\text { Fokus auf persönlichkeitsdefinierende Merkmale und } \\
\text { die zentralen Aspekte einer Szenerie }\end{array}$ & $\begin{array}{l}\text { Fokus auf beziehungsdefinierende Merkmale und den Kontext } \\
\text { einer Szenerie }\end{array}$ \\
\hline & $\begin{array}{l}\text { Der Typ ist kräftig gebaut und macht einen feindseligen } \\
\text { bzw. verächtlichen Eindruck. }\end{array}$ & $\begin{array}{l}\text { Die anderen aus meiner Gruppe haben das Geschehen beobachtet } \\
\text { und sehen mich erwartungsvoll an. }\end{array}$ \\
\hline \multirow[t]{2}{*}{ Interpretieren $^{\mathrm{d}}$} & $\begin{array}{l}\text { Referenz auf internale Attribute, Neigungen und das } \\
\text { Wissen über die eigene Persönlichkeit } \rightarrow \text { eigene Mei- } \\
\text { nung und unterstellte Absichten des anderen sind rele- } \\
\text { vant }\end{array}$ & $\begin{array}{l}\text { Referenz auf externale situationale-kontextuale Attribute und das } \\
\text { Wissen über die Beziehungen zu anderen } \rightarrow \text { erwartete soziale } \\
\text { Normen in der Situation sind relevant }\end{array}$ \\
\hline & $\begin{array}{l}\text { Der Typ will mir schaden, was mich und meine Person } \\
\text { infrage stellt. }\end{array}$ & $\begin{array}{l}\text { Leute wie der wollen meiner Gruppe und ihrem Ansehen schaden; } \\
\text { wenn ich nichts tue, bin ich für diese Schmach verantwortlich. }\end{array}$ \\
\hline \multirow[t]{2}{*}{ Planen $^{\mathrm{e}}$} & $\begin{array}{l}\text { Präferenz von persönlichen Zielen, Wünschen und } \\
\text { Annäherungszielen }\end{array}$ & $\begin{array}{l}\text { Präferenz von Zielen und Wünschen von anderen und Vermei- } \\
\text { dungszielen }\end{array}$ \\
\hline & $\begin{array}{l}\text { Ich muss etwas tun, um meine Überlegenheit zu bewei- } \\
\text { sen. Dafür ist es in Ordnung, andere zu schlagen, weil } \\
\text { das einen deutlichen Erfolg hervorbringt }\end{array}$ & $\begin{array}{l}\text { Ich muss mich verteidigen, um zu zeigen, dass man mit uns nicht } \\
\text { so umspringen kann. Damit übernehme ich Verantwortung. Den } \\
\text { anderen zu schlagen, ist das, was von mir erwartet wird und wofür } \\
\text { ich Respekt bekommen werde. }\end{array}$ \\
\hline \multirow[t]{2}{*}{ Handeln ${ }^{\mathrm{f}}$} & $\begin{array}{l}\text { System der Aktivierung und Initialisierung, um Annä- } \\
\text { herungsziele zu erreichen; aktives Reappraisal als beste } \\
\text { Coping-Strategie }\end{array}$ & $\begin{array}{l}\text { System der Inhibition, Suppression und Regulation, um uner- } \\
\text { wünschte Konsequenzen zu vermeiden; passive Suppression als } \\
\text { beste Coping-Strategie }\end{array}$ \\
\hline & $\begin{array}{l}\text { Ich konzentriere mich auf mein Ziel, den anderen so zu } \\
\text { schlagen, dass es ihn hart trifft. }\end{array}$ & $\begin{array}{l}\text { Ich tue, was ich kann, um mein Ansehen in der Gruppe und das } \\
\text { Ansehen der Gruppe nach außen nicht zu verlieren. }\end{array}$ \\
\hline \multirow[t]{2}{*}{ Reflektieren ${ }^{g}$} & $\begin{array}{l}\text { Persönlicher Erfolg und Selbstwert sind wesentliche } \\
\text { Kriterien; andere fungieren eher als Vergleichsindikato- } \\
\text { ren und als Spiegel }\end{array}$ & $\begin{array}{l}\text { Die Bewertung und Zufriedenheit von anderen sowie das Befinden } \\
\text { der Gruppe als Ganzes sind wesentliche Kriterien }\end{array}$ \\
\hline & $\begin{array}{l}\text { Es war leicht, den anderen zu schlagen, was zeigt, } \\
\text { dass ich überlegen bin und man mit mir so etwas nicht } \\
\text { machen kann. }\end{array}$ & $\begin{array}{l}\text { Dass der Typ sich zurückzieht, zeigt, dass er uns nun respektiert. } \\
\text { Dass ich es geschafft habe, meine Leute zu verteidigen, macht uns } \\
\text { alle froh und bringt mich meiner Gruppe näher. }\end{array}$ \\
\hline
\end{tabular}

Die Komponenten beziehen sich auf die einzelnen Aspekte des Agency-Prozesses; beispielhafte Ausprägungen sind kursiv dargestellt

${ }^{a}$ Markus und Kitayama (1998, 1991, 2010)

${ }^{b}$ Eid und Diener (2009), Kitayama et al. (2006), Mesquita et al. (2016), Uchida und Kitayama (2009), Uchida et al. (2004), Tsai et al. (2006)

${ }^{\mathrm{c}}$ Markus und Kitayama (1991), Masuda et al. (2008, 2012), Miyamoto et al. (2011), Nisbett und Masuda (2003), Nisbett et al. (2001)

${ }^{\mathrm{d}}$ Lee et al. (2015), Na und Kitayama (2011), Norenzayan und Nisbett (2000), Norenzayan et al. (2002)

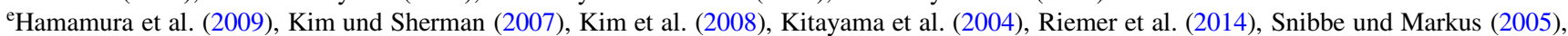

Keltner et al. (2003)

fHiggins et al. (2008), Kim und Lawrie (2018), John und Gross (2004), Matsumoto et al. (2008)

${ }^{\mathrm{g}}$ Markus und Kitayama (1991) 
gene Bestellung aufgibt. Wie je nach Trait bestimmte Informationsverarbeitungsmechanismen eher präferieret werden und wie sich dies in dem Beispiel ausdrücken könnte, ist in Tab. 1 gezeigt. Die Unterschiede zwischen den Verarbeitungsmechanismen ergeben sich aus der kulturpsychologischen bzw. kulturvergleichenden psychologischen Forschung. Sie sind für jeden Trait und jede Komponente im Agency-Prozess einzeln dargestellt. Darunter ist jeweils in kursiv eine mögliche spezifische Ausprägung aus der Sicht des Protagonisten des Beispiels formuliert.

Das Beispiel soll zeigen, dass sich das Verhalten je nach angenommenem kulturellen Trait unterschiedlich erklärt, während die Situation und die Reaktion sich nicht unterscheiden müssen. Diese Unterschiede in der Erklärung hätten gleichfalls Einfluss auf die abgeleiteten Interventionen. Während bei dem unabhängigen Verarbeitungsstil beispielsweise die Auseinandersetzung mit dem eigenen Selbstbild zu empfehlen wäre, könnte jemand mit einem interdependenten Verarbeitungsstil möglicherweise eher von einer die Bezugsgruppe einbeziehenden Straftatauseinandersetzung profitieren.

Die Unterscheidung zwischen unabhängigem vs. interdependentem Verarbeitungsstil bzw. Selbstkonzept wurde gewählt, weil diese Differenzierung zu den prominentesten in der Kulturpsychologie und kulturvergleichenden Psychologie zählt (Greenfield et al. 2003). Sich diese unterschiedlichen Verarbeitungsstile bewusst zu machen, sollte auch mit Blick auf die kulturelle Diversität der Straffälligenpopulation relevant sein. Es kann davon ausgegangen werden, dass die allermeisten Straffälligen, die nicht aus einem euroamerikanischen Kontext stammen, eher einen interdependenten Verarbeitungsstil bevorzugen, während die Beurteiler eher einen unabhängigen Verarbeitungsstil gelernt haben (Hofstede 2001; Schwartz 2006). Das bedeutet jedoch nicht, dass es sich bei diesen verschiedenen Selbstwahrnehmungen um separate, singulär vorkommende und unveränderbare Eigenschaften einer Person handelt (Vignoles et al. 2016). Diese verschiedenen Selbstkonzepte und Verarbeitungsstile sind vielmehr dynamisch, können in unterschiedlichen Situationen unterschiedlich stark aktiviert sein (Oyserman und Lee 2008) und sind von Prozessen der Akkulturation stark beeinflusst (Hong et al. 2000). Darüber hinaus hat eine Person sehr viele kulturelle Traits, die sich mitunter auch widersprechen mögen, internalisiert, und dass das Erlernen bestimmter Traits noch nicht deren Aktivierungsstärke vorhersagt (Schmidt et al. im Druck).

\section{Praktische Implikationen}

Das CAMCB ist v.a. ein Rahmenmodell für die Erklärung von delinquentem Verhalten. Neben dem theoretischen Mehrwert kann die Anwendung eines solchen Modells je- doch auch für die Prognose und Behandlung von Bedeutung sein, wie die Autoren folgend skizzieren werden.

Erklärung Der erste Schritt innerhalb der idiografisch geleiteten Urteilsbildung ist die Erklärung der individuellen Delinquenz, welche in der Formulierung einer individuellen Kriminaltheorie bzw. Delinquenzhypothese mündet (Dahle 2010). Dafür müssen nicht nur die zugrunde liegenden psychologischen Mechanismen delinquenten Handelns im Sinne einer Deliktdynamik beschrieben werden. Es gilt auch, explizit zwischen universellen Komponenten und deren kulturell bzw. individuell gefärbten Ausprägungen zu unterscheiden. Dahle (2010) fordert bereits, dass die Grundlage für die individuelle Kriminaltheorie das Herausarbeiten von charakteristischen Verhaltensmustern, Denkgewohnheiten, Handlungskompetenzen, Interaktionsmustern und überdauernden Bedürfnissen ist. Diese grundlegenden Erlebensund Verhaltensmuster zum Tatzeitpunkt müssten jedoch bereits kultursensibel, d. h. vor dem Hintergrund beeinflussender kultureller Traits, betrachtet werden. Die in dieser Phase des Urteilsprozesses herangezogenen Theorien und empirischen Befunde müssten stets kultursensibel interpretiert werden. Das bedeutet, dass die Anwendung der Grundprinzipien des CAMCB dazu beitragen könnten, dass die individuelle Kriminaltheorie transparenter, kohärenter und kultursensibel ist.

Die im CAMCB spezifizierten universellen Komponenten des Agency-Prozesses lassen sich schablonenartig für die Erklärung der Aktualgenese individueller Delinquenz heranziehen. Welche kulturellen Traits mögen zur Tatzeit aktiviert gewesen sein und was mag diese Aktivierung getriggert haben? Welche Wirkung mögen diese Traits auf die Verhaltensgenese im Allgemeinen und die delinquente Handlung der Anlasstat im Besonderen gehabt haben? Um die Traits zu extrahieren, die den meisten Erklärungswert haben, muss zwischen Verfügbarkeit, Zugänglichkeit und Aktivierung unterschieden werden. Ein Trait ist prinzipiell verfügbar, wenn er abgespeichert wurde. Das bedeutet jedoch nicht, dass dieses Wissen auch leicht abrufbar ist. Das ist dann der Fall, wenn der Trait durch externale oder internale Trigger leicht zugänglich, d.h. aktivierbar, ist. $A k$ tivierung bezieht sich wiederum auf den aktuellen Status. Aktivierung ist dann wahrscheinlicher, wenn der Trait kurz zuvor aktiviert wurde oder häufig aktiviert ist und wenn er zu den Reizen der Situation passt (Oyserman und Yan 2018; Higgins und Kruglanski 1996). Das bedeutet für kulturelle Traits, dass diese einflussreicher sind, wenn der kulturelle Kontext zuvor deutlich hervorgehoben wurde (Chiu et al. 2010). Diese Aktivierung und die Aufrechterhaltung dieser Aktivierung ist gleichfalls von der Motivation des Individuums abhängig (Eitam und Higgins 2010). Wenn ein kultureller Trait von der Person als bedeutsam wahrgenommen wird (Oyserman und Yan 2018), kann dies die Wirkung 
dieses Traits auf den Agency-Prozess erhöhen; eine Befürwortung ist jedoch nicht unbedingt erforderlich ( $\mathrm{Na}$ und Kitayama 2011). Das bedeutet, dass es in der Praxis wesentlich ist, zunächst den grundlegenden Verarbeitungsstil zu erkennen und diesen kulturell und individuell gefärbten Agency-Prozess gut begründet zu beschreiben. Darauf aufbauend könnte dann der Einfluss der mentalen Eindrücke ergründet werden, die die Tathandlung mitbedingt haben mögen. Welche mentalen Eindrücke mögen für die Tathandlung von Bedeutung gewesen sein, und wie mögen diese vor dem Hintergrund des kulturell-individuellen Agency-Prozesses verarbeitet worden sein? Welche Cues mögen diese Eindrücke getriggert haben? Worin mögen die Ursachen der Delinquenz liegen. Handelt es sich um eingeschränkte Funktionsweisen universeller Verhaltenskomponenten, maladaptive mentale Eindrücke oder die delinquenzbegünstigende Verarbeitung sozial adaptiver mentaler Eindrücke? Wie intensiv bzw. konsistent mag dies auf die Verhaltensgenese gewirkt haben?

Mit Blick auf die hier gewählten kulturellen Traits (unabhängiger vs. interdependenter Verarbeitungsstil) lässt sich vermuten, dass die Ursachen für die Delinquenz schwerpunktmäßig in verschiedenen Bereichen zu suchen wären. Bei einem unabhängigen Verarbeitungsstil sollten Persönlichkeitseigenschaften eine zentralere Rolle bei der Erklärung der Delinquenz einnehmen als bei einem interdependenten Verarbeitungsstil, bei dem soziale und kontextuale Variablen stärker im Fokus stehen sollten (Dwairy 2002).

Prognose Für die Vorhersage von Verhalten mag das CAMCB insofern eine Rolle spielen, als dass die in der individuellen Kriminaltheorie spezifizierten Mechanismen hinsichtlich ihrer Stabilität (zeitlich und kontextabhängig) bewertet werden (Dahle 2010). Eine zeitlich relativ stabile Einschränkung der universellen Funktionsweise des Agency-Prozesses, die ursächlich für die Delinquenz gewesen sein mag, wird sicherlich eine prognostisch ungünstigere Bewertung zur Folge haben als ein maladaptiv verarbeiteter mentaler Eindruck, welcher durch seltene äußere Reize getriggert wurde. Hinsichtlich maladaptiv wirkender mentaler Eindrücke wird auch die Frage nach deren genereller Zugänglichkeit und konsistenter Wirkung auf verschiedene Komponenten des Agency-Prozesses von Bedeutung sein. Wenn alle Schritte im Agency-Prozess kohärent sind, d.h., sich gegenseitig stringent bedingen, ist anzunehmen, dass die mentalen Eindrücke schon vor der Handlung leicht zugänglich waren. Es ist wohl davon auszugehen, dass durch die Verstärkung infolge der Handlung diese Eindrücke auch in Zukunft leicht zugänglich und damit handlungsleitend sein werden.

Mit Blick auf die beispielhaft gewählten kulturellen Traits sollte die Verhaltensvorhersage für einen unabhängigen Verarbeitungsstil leichter fallen als für einen interde- pendenten Verarbeitungsstil. Da Interdependenz durch eine hohe Adaptivität an den jeweiligen Kontext charakterisiert ist, ist die Vorhersage generell unsicherer, und prognostische Aussagen müssten den jeweiligen Kontext, auf den sie sich beziehen, genau spezifizieren.

Behandlung Die im CAMCB vorgesehene Bewertung hinsichtlich Funktionalität, Adaptivität und Legalität des Verhaltens hat auch für die Behandlungsplanung einige Vorteile. Gängige Behandlungsprogramme orientieren sich häufig an der Art des Gesetzesverstoßes (z.B. Behandlungsprogramme für Sexualstraftäter), welches eine wenig delinquenzerklärende, aber sehr verbreitete Klassifikation in der Kriminalpsychologie ist (Ward und Carter 2019). Werden Interventionen aber an den tatsächlichen Ursachen der Delinquenz orientiert, sollte nicht nur die rückfallreduzierende Wirkung besser sein; die Behandlungsmaßnahmen könnten auch besser evaluiert werden (Heffernan et al. 2019). GroBe Unterschiede in der Behandlungsmethode und Intensität sollten sich dabei v. a. durch die Differenzierung von übergreifenden Dysfunktionalitäten vs. maladaptiven Schemata ergeben. Eine gute Beschreibung des kulturell und individuell gefärbten Agency-Prozesses liefert wiederum wichtige Hinweise zu der Art und Weise der Behandlung (s. ,responsivity principle“ von Andrews und Bonta 2010). Demnach wäre eine personenzentrierte Behandlung, die an den individuellen Einstellungen und Bedürfnissen ansetzt, eher für einen unabhängigen Verarbeitungsstil geeignet. Bei einem interdependenten Verarbeitungsstil müssten die sozialen Bezüge viel stärker einbezogen werden, was systemische Behandlungsansätze eher ermöglichen.

Dadurch, dass sich das CAMCB auf grundlegende psychologische Prozesse bezieht, erklärt es delinquentes Verhalten mit den gleichen Bedürfnissen und Mechanismen wie jedes andere Verhalten auch. Dies unterstützt eine Ressourcenorientierung (z. B. im Rahmen des Good Lives Model), die essenziell für das Commitment und die Motivation während einer Behandlung ist (Ward und Stewart 2003). Ein solcher Perspektivwechsel erscheint besonders bei der ohnehin schon stark stigmatisierten Gruppe Straffälliger mit diverser kultureller Herkunft von großer Bedeutung zu sein (Jones et al. 2002; von Gostomski 2003; Shepherd und Lewis-Fernandez 2016).

Erfassung kultureller Traits Weil im CAMCB auf die zugrunde liegenden psychologischen Mechanismen abgehoben wird und kulturelle Traits als eine Form mentaler Eindrücke aufgefasst werden, ist es auch nicht nötig, die Ethnie oder das Herkunftsland einer Person zu kennen (Bond und van de Vijver 2011). Nur die Aktivierung und Befürwortung kultureller Traits ist für die Erklärung von Verhalten wichtig. Eine diskriminierende und stereotypengeleitete Vorgehensweise bei der Auswahl der spezifischen Theo- 
rie ist demnach weniger wahrscheinlich. Dies setzt allerdings voraus, dass zuvor bestimmt wurde, welche kulturellen Traits am einflussreichsten gewesen sein mögen. Hierbei sollte zwischen eher übergreifenden kulturellen Traits (z.B. unabhängiges Selbstkonzept) und spezifischeren kulturellen Traits (z.B. bestimmte kulturelle Rituale) unterschieden werden. Während sich spezifische kulturelle Traits wohl eher situationsgebunden aufdrängen, sollten übergreifende kulturelle Traits immer im Blick behalten werden, weil sie den Agency-Prozess als solchen tiefgreifend beeinflussen. Deshalb erscheint eine der eigentlichen Exploration vorgelagerte Befragung (Dwairy 2002) oder standardisierte Erhebung (z.B. Singelis 1994; Singelis et al. 1995; Kitayama et al. 2009) der präferierten kulturellen Traits sinnvoll. Um diese Informationen fachgerecht zu erfassen und entsprechend einfließen lassen zu können, wäre es für Praktiker wesentlich, dass entsprechendes Wissen und Handlungskompetenzen (z. B. in interkulturellen Kompetenztrainings) fortgebildet werden (Schmidt 2018).

\section{Diskussion}

Eine hohe kulturelle Diversität gehört zum Alltag in der Arbeit mit Straffälligen, was eine ganze Reihe zusätzlicher Phänomene und Herausforderungen mit sich bringt (Dahle et al. 2020). Diesen Herausforderungen ist mit gängigen Risikofaktoren und Erklärungsmodellen von Delinquenz kaum zu begegnen, da erhebliche Zweifel an deren allgemeiner Gültigkeit im interkulturellen Kontext bestehen (Put et al. 2013; Shepherd und Lewis-Fernandez 2016; Jones et al. 2002; Hart 2016; Schmidt et al. 2020). Auch die Entwicklung eigenständiger Theorien und Prognoseinstrumente für einzelne kulturelle Kontexte erscheint vor dem Hintergrund einer zunehmenden kulturellen Pluralisierung unzulänglich und mit Blick auf etwaige Auswahlverfahren diskriminierend (Schmidt 2018). Wünschenswert wäre vielmehr eine Herangehensweise, die ohne diskriminierende Kategorisierungen auskommt und flexibel gegenüber den sich ständig ändernden gesellschaftlichen Lebensbedingungen und der vielfältigen kulturellen Diversität ist. Mit dem Cultural Agency-Model of Criminal Behavior (CAMCB) haben wir ein Rahmenmodell vorgeschlagen, das auf die zugrunde liegenden individuellen psychologischen Mechanismen delinquenten Handelns abhebt und systematisch zwischen universellen Komponenten und deren kulturell-individuell gefärbten Ausprägungen unterscheidet. Delinquentes Verhalten kann damit unter der Verwendung ganz verschiedener kultureller Linsen erklärt werden. Dadurch ist es auch möglich, Unterschiede in der Ausprägung verschiedener Risikofaktoren zu erklären. Beispielsweise wirken kriminogene Einstellungen, wie sie Andrews und Bonta (2010) definiert haben, bei Individuen, die kulturell bedingt höchstwahrscheinlich einen eher unabhängigen Verarbeitungsstil haben (z. B. deutsche Täter ohne Migrationshintergrund), stärker auf Delinquenz als bei Individuen, die höchstwahrscheinlich einen eher interdependenten Verarbeitungsstil priorisieren (z. B. Täter mit arabischem Migrationshintergrund; Dahle und Schmidt 2014; Schmidt et al. 2018; Shepherd et al. 2015). Diese Einstellungen fokussieren auf individuelle Überzeugungen, was v. a. bei einem unabhängigen Verarbeitungsstil intentionsbildend und handlungsleitend sein sollte (Markus 2016).

Im CAMCB wird der Einfluss der kulturellen Sozialisation dabei nicht an eine geografische oder ethnische Herkunft gebunden, sondern an kulturelle Traits (z.B. interdependenter Verarbeitungsstil), welche die Verhaltensgenese beeinflussen. Das Rahmenmodell zeigt, wie unter der Annahme bestimmter kultureller Traits eine weiterführende spezifischere Theorieformulierung stattfinden kann. Dies erscheint nicht nur für die kriminalpsychologische Theorieentwicklung von Bedeutung, sondern auch für die forensische Praxis, bei der für jeden Einzelfall eine Theorie über dessen individuelle Delinquenz formuliert werden muss.

\section{Vorläufige Evaluation}

Wenngleich es sich beim CAMCB lediglich um ein Rahmenmodell handelt, was für bestimmte Kontexte und Fragestellungen eine Grundlage für spezifische Theorien bilden kann, lässt sich dieses Rahmenmodell mit allgemeinen Gütekriterien für Theorien beurteilen. Im Folgenden wird für eine vorläufige Evaluation des Modells auf die Kriterien von Popper (1989) und Thagard (1989) in einer vereinfachten Darstellung zurückgegriffen.

Sparsamkeit Das CAMCB kommt mit relativ wenigen Annahmen aus, die gleichfalls nicht an spezifische kulturelle Normen gebunden sind. Im Grunde ist die Idee, die hinter dem CAMCB steckt, relativ einfach: 1) Identifikation von universellen Verhaltenskomponenten; 2) Identifikation und Beschreibung von relevanten kulturbedingten Einflüssen auf diese universellen Aspekte; 3) Bewertung des Verhaltens und der zugrunde liegenden Komponenten. Damit wird jede zusätzliche Annahme (z.B. welche kulturellen Traits bedeutsam sind) transparent formuliert.

Erschöpfungsgrad Dieser sehr simple Grundgedanke einer dreistufigen Erklärung lässt sich nicht nur auf Theorien zur Aktualgenese von Verhalten anwenden, so wie durch das CAMCB vorgeschlagen, sondern auch auf entwicklungs- und persönlichkeitspsychologische Theorien. Denn auch Entwicklungstheorien beschreiben die Manifestation universeller Entwicklungsprozesse (z.B. Übergang zum Erwachsenenalter) in einem kulturellen und sozialen Kontext (z.B. individualistisch geprägt) und stellen Kriterien 
zur Beurteilung der Entwicklung (z. B. Ablösung vom Elternhaus) bereit. Diese Grundidee des CAMCB findet sich schon bei Brandtstädter (1990; zit. nach Dahle et al. 2020), der davon ausgeht, dass die Entwicklung eines Menschen (1) durch lebensaltersgebundene Entwicklungsprozesse, (2) durch individuelle Lebensereignisse und (3) durch kontextuelle (z.B. kulturelle) Einflüsse gesteuert wird. Werden diese einzelnen Aspekte sauber voneinander getrennt und aufeinander aufbauend beschrieben, wäre es möglich, zu einer kohärenten, kultursensiblen Erklärung von Entwicklungsverläufen zu kommen. Der Erschöpfungsgrad des CAMCB ist aber nicht nur durch den simplen Grundgedanken hoch, sondern auch, weil die Autoren auf grundlagenpsychologische Konzepte zurückgreifen. Die Verwendung grundlagenpsychologischer Konzepte stellt Anknüpfungspunkte für andere Erklärungsebenen (z.B. neurologisch, entwicklungspsychologisch) bereit, da diese Konzepte auch von diesen Perspektiven aufgegriffen werden. Dies ermöglicht eine pluralistische Erklärung von Delinquenz, die in Anbetracht der Heterogenität und Komplexität dieses Phänomens angemessener erscheint (Ward et al. im Druck). Dass die Konzepte ohne eine vorgegebene normative Wertung (legal vs. illegal; normal vs. abnormal) auskommen, hat den Vorteil, dass Wissen über spezifische (und dabei ganz verschiedene) kulturelle Kontexte oder individuelle Besonderheiten gezielt in die Erklärung eingehen kann (Schmidt et al. im Druck). Das CAMCB kann aber schon ohne weiterführende Spezifikation erklären, warum bestimmte gängige Risikofaktoren in diversen kulturellen Kontexten anders ausgeprägt sind bzw. in anderer Weise mit Delinquenz im Zusammenhang stehen. Dies ist mit den bisherigen Theorien, die nur für einen euroamerikanischen Kontext formuliert wurden, nicht konsistent möglich.

Widerspruchsfreiheit Das CAMCB ist sehr nahe an bereits bekannten und teils gut etablierten Theorien (Crick und Dodge 1994; Lemerise und Arsenio 2000; Heffernan und Ward 2017; Anderson und Bushman 2002; Brandtstädter und Rothermund 2002; Wyer 2012; Eitam und Higgins 2010; Wikstrom und Treiber 2007; Wikstrom 2009) formuliert worden. Auch die Herleitung von unterschiedlichen Verarbeitungsmechanismen je nach kulturellem Trait gründet sich auf einer breiten empirischen Grundlage (dazu Schmidt et al. im Druck). Damit lässt sich von einer relativ hohen äußeren Widerspruchsfreiheit ausgehen. Darüber hinaus sollte das schrittweise Vorgehen bei der Erklärung von Delinquenz, welches einer axiomatischen Theorieformulierung ähnlich ist, auch Inkonsistenzen innerhalb der Theorie reduzieren und die Transparenz der Erklärung erhöhen.

Überprüfbarkeit Es sollte möglich sein, für jede Hypothese, die aus dem CAMCB abgeleitet wird, eine gegenteilige Beobachtung zu formulieren, die diese Hypothese wi- derlegen würde (Popper 1989). Beispielsweise würde eine egoistische Motivwahl bei einer Person mit einem nachweislich aktivierten interdependenten Verarbeitungsmechanismus unseren Annahmen widersprechen. Einschränkend muss jedoch erwähnt werden, dass eine empirische Prüfung und somit wichtigstes Gütemerkmal einer Theorie bislang noch nicht umfassend stattfand. Beim CAMCB handelt es sich um ein Rahmenmodell; der dezidierten Prüfung müssen zunächst eine konkretere Theorie- und Hypothesenbildung sowie die Festlegung der Messkonzepte und Operationalisierungen vorangehen.

\section{Ausblick}

Theoretische Weiterentwicklung Das CAMCB ist in der vorliegenden Form noch recht unspezifisch und könnte in Zukunft in viele Richtungen erweitert werden. Zuvorderst müssten die einzelnen Komponenten und Prozesse jeweils detaillierter beschrieben werden. Dies wäre beispielsweise mittels etablierter Theorien zu Intentionsbildung (z.B. Ajzen 1991) möglich. Auch eine stärkere Spezifikation der kulturellen Traits wäre wünschenswert. Wie ließen sich die verschiedenen Variationen der kulturellen Traits, die innerhalb eines Individuums koexistieren können (Vignoles et al. 2016), in dem Modell integrieren? Es ließe sich auch einwenden, dass sich das CAMCB durch die Anlehnung an die Arbeiten von Crick und Dodge (1994) ausschließlich auf aggressives Verhalten bezieht. Es wäre in Zukunft nötig, theoretisch zu ergründen, ob sich das Modell genauso auf nichtgewalttätige Delinquenz anwenden lässt bzw. welche Anpassungen vorgenommen werden müssten. Darüber hinaus wäre auch eine Spezifikation von protektiv wirkenden Einflüssen wichtig, um eine ressourcenorientierte Perspektive stärker zu unterstützen. Es müsste noch genauer definiert werden, was vor dem Hintergrund des CAMCB als Schutzfaktoren betrachtet werden kann und nicht allein die Abwesenheit eines Risikos ist (Ward 2017).

Empirische Forschung Die zahlreichen Hypothesen über die Genese delinquenten Verhaltens, die sich aus dem systematischen Einbezug unterschiedlicher kultureller Traits ergeben (s. Beispiel), sollten in Zukunft gezielt überprüft werden. Diese dezidierte Ableitung von Hypothesen und Überprüfung dieser ließe sich dann auch auf eine ganze Reihe weiterer kultureller Traits (z.B. „high honour“ vs. „low honour“, Cohen et al. 1996; ,tight“"vs. ,loose culture“, Gelfand et al. 2006) und deren Zusammenspiel ausweiten. Bei der Überprüfung solcher Hypothesen erscheint zunächst die Fokussierung auf einzelne Teilannahmen sinnvoll. So ließen sich beispielsweise Paradigmen im Kontext der ,Theory of Planned Behavior“ (Ajzen 1991) heranziehen, um die variablen Einflüsse von sozialen Werten und dem Selbstbild auf die Intentionsbildung zu untersuchen. Um den Ein- 
fluss des kulturellen Trait auf verschiedene Komponenten delinquenten Verhaltens zu analysieren, wären nicht nur quasiexperimentelle Zuordnungen auf Basis einer selbsteingeschätzten Präferenz für einzelne Traits in Erwägung zu ziehen. Vor allem experimentelle Manipulationen über Priming (Oyserman und Lee 2008) könnten dazu beitragen, dass der Einfluss von situationalen Variablen auf den Agency-Prozess allgemein und die Genese einer delinquenten Handlung im Spezifischen besser aufgeklärt werden kann. Interessant wäre auch, ob innerhalb einer Handlungssequenz unterschiedliche Traits (z.B. Unabhängigkeit vs. Interdependenz) bei unterschiedlichen Komponenten des Agency-Prozesses aktiviert sein können (z.B. eine vom unabhängigen Trait gesteuerte Interpretation mit einer vom interdependenten Trait gesteuerten Intentionsbildung) und welchen Einfluss dies auf die (delinquenzbegünstigende) Handlungsentscheidung hat. Operationalisierungen und experimentelle Manipulation, die sich in solchen Untersuchungen bewähren, könnten auch in einzelfallexperimentelle Methoden (Manolov et al. 2014) überführt und bei der Diagnostik in der Praxis eingesetzt werden. Damit könnte langfristig überprüft werden, ob eine Erklärung, die auf dem CAMCB fußt, auch für Prognose und Behandlungsplanung valide Aussagen treffen kann. Parallel dazu scheint die Untersuchung von Entwicklungsverläufen für die empirische Forschung zum CAMCB von großer Bedeutung zu sein. Wie entstehen im Individuum verankerte und zugängliche Traits (e.g., Sahin-Acar und Leichtman 2015) und wie interagieren sie mit Variablen der sozialen Interaktionen, Institutionen und verschiedenen politischökonomischen Kontexten (Hamedani und Markus 2019; Henrich 2020)? Darauf aufbauend ließe sich analysieren, welche Entwicklungsbedingungen in den verschiedenen kulturellen Kontexten dazu führen, dass bestimmte mentale Eindrücke besonders einfach zugänglich und damit häufiger handlungsleitend für Delinquenz sind als andere (Dahle et al. 2020). Dies ließe sich einerseits über eine Mischung aus retrospektiver Fragebogenerhebung von Entwicklungsbedingungen und der szenarienbasierten Erhebung von Verarbeitungsmechanismen relevanter Situationen (Crick und Dodge 1994) mithilfe psychophysischer Parameter (Horsley et al. 2010) umsetzen. Andererseits könnten auch Längsschnittstudien durchgeführt werden, die zu verschiedenen Messzeitpunkten diese Verarbeitungsmechanismen erheben und in Bezug zu parallel gemessenen Entwicklungsparametern setzen. Insgesamt scheinen für solche Untersuchungen Mehrebenenanalysen (Bond und van de Vijver 2011) sinnvoll, weil diese ermöglichen, die verschiedenen Variablen der unterschiedlichen Erklärungsebenen zu differenzieren, um sich damit einer pluralistischen Erklärung (Ward et al. im Druck) anzunähern.
Danksagung Dieses Projekt wurde durch ein Stipendium des Deutschen Akademischen Austauschdienstes (DAAD) für Postdoktoranden an die Erstautorin möglich. Gedankt sei ganz besonders Dr. Roxanne Heffernan für ihre unverzichtbare und wichtige Arbeit in diesem Projekt. Prof. Dr. Taciano Milfont danken wir für seine vielen Inspirationen und wertvollen Hinweise, die unsere Arbeit sehr bereichert haben.

Funding Open Access funding enabled and organized by Projekt DEAL.

Interessenkonflikt S. Schmidt und T. Ward geben an, dass kein Interessenkonflikt besteht.

Open Access Dieser Artikel wird unter der Creative Commons Namensnennung 4.0 International Lizenz veröffentlicht, welche die Nutzung, Vervielfältigung, Bearbeitung, Verbreitung und Wiedergabe in jeglichem Medium und Format erlaubt, sofern Sie den/die ursprünglichen Autor(en) und die Quelle ordnungsgemäß nennen, einen Link zur Creative Commons Lizenz beifügen und angeben, ob Änderungen vorgenommen wurden.

Die in diesem Artikel enthaltenen Bilder und sonstiges Drittmaterial unterliegen ebenfalls der genannten Creative Commons Lizenz, sofern sich aus der Abbildungslegende nichts anderes ergibt. Sofern das betreffende Material nicht unter der genannten Creative Commons Lizenz steht und die betreffende Handlung nicht nach gesetzlichen Vorschriften erlaubt ist, ist für die oben aufgeführten Weiterverwendungen des Materials die Einwilligung des jeweiligen Rechteinhabers einzuholen.

Weitere Details zur Lizenz entnehmen Sie bitte der Lizenzinformation auf http://creativecommons.org/licenses/by/4.0/deed.de.

\section{Literatur}

Aebi M, Tiago M, Burkhardt C et al (2015) SPACE I-council of europe annual penal statistics: prison populations; survey 2014. Council of Europe, Strasbourg

Agnew R (1992) Foundation for a general strain theory of crime and delinqunency. Criminology 30:47-88. https://doi.org/10.1111/j. 1745-9125.1992.tb01093.x

Ajzen I (1991) The theory of planned behavior. Organ Behav Human Decis Proc 50:179-211

Anderson CA, Bushman BJ (2002) Human aggression. Annu Rev Psychol 53:27-51. https://doi.org/10.1146/annurev.psych.53.100901. 135231

Andrews DA, Bonta J (1995) LSI-R; The level of service inventoryrevised user's manual. Multi-Health Systems, Toronto

Andrews DA, Bonta J (2010) The psychology of criminal conduct. Anderson Publishing, Cincinnati

Bandura A (2006) Toward a psychology of human agency. Perspect Psychol Sci 1:164-180. https://doi.org/10.1111/j.1745-6916. 2006.00011.x

Berry JW, Poortinga YH, Breugelmans SM, Chasiotis A, Sam DL (2011) Cross-cultural psychology; research and applications. Cambridge University Press, Cambridge, New York

Bond MH, van de Vijver FJR (2011) Making scientific sense of cultural differences in psychological outcomes:; Unpackaging the Magnum Mysterium. In: Matsumoto DR, van de Vijver, Fons JR (Hrsg) Cross-cultural research methods in psychology. Cambridge University Press, Cambridge, S 75-100

Bonta J, Andrews DA (2017) The psychology of criminal conduct. Routledge Taylor \& Francis Group, London, New York

Brandtstädter J, Rothermund K (2002) The life-course dynamics of goal pursuit and goal adjustment: a two-process framework. Dev Rev 22:117-150. https://doi.org/10.1006/drev.2001.0539 
Chiu C-Y, Hong Y-Y (2007) Cultural processes: basic principles. In: Kruglanski AW, Higgins ET (Hrsg) Social psychology. Handbook of basic principles. Guilford, New York, London, S 785-804

Chiu C-Y, Gelfand MJ, Yamagishi T, Shtenynberg G, Wan C (2010) Intersubjective Culture; The role of intersubjective perceptions in cross-cultural psychology. Perspect Psychol Sci 5:482-493

Cohen D, Nisbett RE, Bowdle BF, Schwarz N (1996) Insult, aggression, and the southern culture of honor: an "experimental ethnography.”. J Pers Soc Psychol 70:945-960. https://doi.org/10.1037/ 0022-3514.70.5.945

Cooper R (2013) Natural kinds. In: Davies M, Graham G, Sadler J, Stanghellini G, Thornton T (Hrsg) The Oxford handbook of philosophy and psychiatry. Oxford University Press, Oxford, S 950-964

Crick NR, Dodge KA (1994) A review and reformulation of social information-processing mechanisms in children's social adjustment. Psychol Bull 115:74-101

v. d PC, Stams G-J, Dekovic M, Hoeve M, v. d LP (2013) Ethnic differences in offense patterns and the prevalence and impact of risk factors for recidivism. Int Crim Justice Rev 23:113-131. https:// doi.org/10.1177/1057567713482940

Dahle K-P (2010) Die Begutachtung der Gefährlichkeit- und Kriminalprognose des Rechtsbrechers. In: Volbert R, Dahle K-P (Hrsg) Forensisch-psychologische Diagnostik im Strafverfahren. Hogrefe, Göttingen, S 67-114

Dahle K-P, Schmidt S (2014) Prognostische Validität des Level of Service Inventory-Revised. Forens Psychiatr Psychol Kriminol 8:104-115. https://doi.org/10.1007/s11757-014-0256-5

Dahle K-P, Greve W, Hosser D, Bliesener T (2020) Das Gefängnis als Entwicklungsraum. Forens Psychiatr Psychol Kriminol 14:3-21. https://doi.org/10.1007/s11757-019-00569-w

Dwairy M (2002) Foundations of psychosocial dynamic personality theory of collective people. Clin Psychol Rev 22:343-360. https:// doi.org/10.1016/S0272-7358(01)00100-3

Eid M, Diener E (2009) Norms for experiencing emotions in different cultures: inter- and Intranational differences. In: Michalos AC, Diener E (Hrsg) Culture and well-being. Springer Netherlands, Dordrecht, S 169-202

Eitam B, Higgins ET (2010) Motivation in mental accessibility: relevance of a representation (ROAR) as a new framework. Social Pers Psych Compass 4:951-967. https://doi.org/10.1111/j.17519004.2010.00309.x

Farrington DP (2020) The integrated cognitive antisocial potential (ICAP) theory: past, present, and future. J Dev Life Course Criminology 6:172-187. https://doi.org/10.1007/s40865-01900112-9

Fortune C-A, Heffernan R (2018) The psychology of criminal conduct: a consideration of strengths, weaknesses and future directions. Psychol Crime Law 5:1-16. https://doi.org/10.1080/1068316X. 2018.1560445

Gelfand MJ, Nishii LH, Raver JL (2006) On the nature and importance of cultural tightness-looseness. J Appl Psychol 91:1225-1244. https://doi.org/10.1037/0021-9010.91.6.1225

Del Giudice M (2018) Evolutionary psychopathology; A unified approach. Oxford University Press, New York

von Gostomski CB (2003) Gewalt als Reaktion auf Anerkennungsdefizite? Köln Z Soziol 55:253-277. https://doi.org/10.1007/s11577003-0050-4

Gottfredson SD, Moriarty LJ (2006) Statistical risk assessment: old problems and new applications. Crime Delinquency 52:178-200. https://doi.org/10.1177/0011128705281748

Greenfield PM, Keller H, Fuligni A, Maynard A (2003) Cultural pathways through universal development. Annu Rev Psychol 54:461-490. https://doi.org/10.1146/annurev.psych.54.101601. 145221

Hamamura T, Meijer Z, Heine SJ, Kamaya K, Hori I (2009) Approach-avoidance motivation and information processing: a cross-cultural analysis. Pers Soc Psychol Bull 35:454-462. https://doi.org/10.1177/0146167208329512

Hamedani MYG, Markus HR (2019) Understanding culture clashes and catalyzing change: a culture cycle approach. Front Psychol 10:700. https://doi.org/10.3389/fpsyg.2019.00700

Hart SD (2016) Culture and violence risk assessment; The case of Ewert v. Canada. J Threat Assess Manag 3:76-96. https://doi.org/ $10.1037 / \operatorname{tam} 0000068$

Heffernan R, Ward T (2017) A comprehensive theory of dynamic risk and protective factors. Aggress Violent Behav 37:129-141. https://doi.org/10.1016/j.avb.2017.10.003

Heffernan R, Wegerhoff D, Ward T (2019) Dynamic risk factors: conceptualization, measurement, and evidence. Aggress Violent Behav 48:6-16. https://doi.org/10.1016/j.avb.2019.06.004

Henrich JP (2020) Weirdest people in the world; How the West became psychologically peculiar and particularly prosperous. Allen Lane, London

Higgins ET, Kruglanski AW (Hrsg) (1996) Social psychology; Handbook of basic principles. Guilford, New York

Higgins ET, Pierro A, Kruglanski AW (2008) Re-thinking culture and personality handbook of motivation and cognition across cultures. Elsevier, Cambridge, USA, S 161-190

Hofstede GH (2001) Culture's consequences; Comparing values, behaviors, institutions, and organizations across nations. SAGE, Thousand Oaks

Hong Y-Y, Morris MW, Chiu C-Y, Benet-Martínez V (2000) Multicultural minds: a dynamic constructivist approach to culture and cognition. Am Psychol 55:709-720. https://doi.org/10.1037/0003066X.55.7.709

Horsley TA, de Castro BO, van der Schoot M (2010) In the eye of the beholder: eye-tracking assessment of social information processing in aggressive behavior. J Abnorm Child Psychol 38:587-599. https://doi.org/10.1007/s10802-009-9361-x

John OP, Gross JJ (2004) Healthy and Unhealthy Emotion Regulation: Personality Processes, Individual Differences, and Life Span Development. J Personality 72 (6):1301-1334

Jones R, Masters M, Griffiths A, Moulday N (2002) Culturally relevant assessment of indigenous offenders: a literature review. Aust Psychol 37:187-197. https://doi.org/10.1080/00050060210001706866

Keltner D, Gruenfeld DH, Anderson C (2003) Power, approach, and inhibition. Psychol Rev 110:265-284. https://doi.org/10.1037/ 0033-295X.110.2.265

Kim HS, Lawrie SI (2018) Culture and motivation. In: Cohen D, Kitayama S (Hrsg) Handbook of cultural psychology, 2. Aufl. Guilford, New York, S 268-291

Kim HS, Sherman DK (2007) "Express yourself": culture and the effect of self-expression on choice. J Pers Soc Psychol 92:1-11. https://doi.org/10.1037/0022-3514.92.1.1

Kim HS, Sherman DK, Taylor SE (2008) Culture and social support. Am Psychol 63:518-526. https://doi.org/10.1037/0003-066X

Kitayama S, Mesquita B, Karasawa M (2006) Cultural affordances and emotional experience: socially engaging and disengaging emotions in Japan and the United States. J Pers Soc Psychol 91:890-903. https://doi.org/10.1037/0022-3514.91.5.890

Kitayama S, Park H, Sevincer AT, Karasawa M, Uskul AK (2009) A cultural task analysis of implicit independence: comparing North America, Western Europe, and East Asia. J Pers Soc Psychol 97:236-255. https://doi.org/10.1037/a0015999

Kitayama S, Snibbe AC, Markus HR, Suzuki T (2004) Is there any "free" choice? Psychol Sci 15:527-533. https://doi.org/10.1111/j. 0956-7976.2004.00714.x

Lee H, Shimizu Y, Uleman JS (2015) Cultural differences in the automaticity of elemental impression formation. Soc Cogn 33:1-19. https://doi.org/10.1521/soco.2015.33.1.1

Lemerise EA, Arsenio WF (2000) An integrated model of emotion processes and cognition in social information processing. Child Dev 71:107-118. https://doi.org/10.1111/1467-8624.00124 
Loeber R, Burke JD (2011) Developmental pathways in juvenile externalizing and internalizing problems. J Res Adolesc 21:34-46. https://doi.org/10.1111/j.1532-7795.2010.00713.x

Lösel F, Bliesener T (2003) Agression und Delinquenz unter Jugendlichen; Untersuchungen von kognitiven und sozialen Bedingungen. Luchterhand, Neuwied

Maiese M (2015) Embodied selves and divided minds. Oxford University Press, Oxford

Manolov R, Gast DL, Perdices M, Evans JJ (2014) Single-case experimental designs: reflections on conduct and analysis. Neuropsychol Rehabil 24:634-660. https://doi.org/10.1080/09602011. 2014.903199

Markus HR (2016) What moves people to action? Culture and motivation. Curr Opin Psychol 8:161-166. https://doi.org/10.1016/j. copsyc.2015.10.028

Markus HR, Kitayama S (1991) Culture and the Self; Implications for cognition, emotion, and motivation. Psychol Rev 98:224-253

Markus HR, Kitayama S (1998) The cultural psychology of personality. J Cross Cult Psychol 29:63-87. https://doi.org/10.1177/ 0022022198291004

Markus HR, Kitayama S (2010) Cultures and selves: a cycle of mutual constitution. Perspect Psychol Sci 5:420-430. https://doi.org/10. $1177 / 1745691610375557$

Masuda T, Ellsworth PC, Mesquita B, Leu J, Tanida S, van de Veerdonk E (2008) Placing the face in context: cultural differences in the perception of facial emotion. J Pers Soc Psychol 94:365-381. https://doi.org/10.1037/0022-3514.94.3.365

Masuda T, Wang H, Ishii K, Ito K (2012) Do surrounding figures' emotions affect judgment of the target figure's emotion? Comparing the eye-movement patterns of European Canadians, Asian Canadians, Asian international students, and Japanese. Front Integr Neurosci 6:72. https://doi.org/10.3389/fnint.2012.00072

Matsumoto D (2007) Culture, context, and behavior. J Personality 75:1285-1319. https://doi.org/10.1111/j.1467-6494.2007.00476. $\mathrm{x}$

Matsumoto D, Yoo SH, Nakagawa S (2008) Culture, emotion regulation, and adjustment. J Personality Soc Psychol 94 (6):925-937

Mesquita B, Boiger M, de Leersnyder J (2016) The cultural construction of emotions. Curr Opin Psychol 8:31-36. https://doi.org/10. 1016/j.copsyc.2015.09.015

Miyamoto Y, Yoshikawa S, Kitayama S (2011) Feature and configuration in face processing: Japanese are more configural than Americans. Cogn Sci 35:563-574. https://doi.org/10.1111/j.1551-6709. 2010.01163.x

Na J, Kitayama S (2011) Spontaneous trait inference is culture-specific: behavioral and neural evidence. Psychol Sci 22:1025-1032. https://doi.org/10.1177/0956797611414727

Nisbett RE, Masuda T (2003) Culture and point of view. Proc Natl Acad Sci 100:11163-11170. https://doi.org/10.1073/pnas. 1934527100

Nisbett RE, Peng K, Choi I, Norenzayan A (2001) Culture and systems of thought: holistic versus analytic cognition. Psychol Rev 108:291-310. https://doi.org/10.1037/0033-295X.108.2.291

Norenzayan A, Nisbett RE (2000) Culture and causal cognition. Curr Dir Psychol Sci 9:132-135. https://doi.org/10.1111/1467-8721. 00077

Norenzayan A, Choi I, Nisbett RE (2002) Cultural similarities and differences in social inference: evidence from behavioral predictions and lay theories of behavior. Pers Soc Psychol Bull 28:109-120. https://doi.org/10.1177/0146167202281010

Olver ME, Stockdale KC, Wormith JS (2014) Thirty years of research on the Level of Service Scales: a meta-analytic examination of predictive accuracy and sources of variability. Psychol Assess 26:156-176. https://doi.org/10.1037/a0035080

Oyserman D, Lee SWS (2008) Does culture influence what and how we think? Effects of priming individualism and collectivism. Psychol Bull 134:311-342. https://doi.org/10.1037/0033-2909.134. 2.311
Oyserman D, Yan VX (2018) Making meaning:; culture-as-situatedcognition approach to the consequences of cultural fluency and Disfluency. In: Cohen D, Kitayama S (Hrsg) Handbook of cultural psychology, 2. Aufl. Guilford, New York, S 536-565

Popper KR (1989) Logik der Forschung. Mohr, Tübingen

Raaijmakers MAJ, Smidts DP, Sergeant JA, Maassen GH, Posthumus JA, van Engeland H, Matthys W (2008) Executive functions in preschool children with aggressive behavior: impairments in inhibitory control. J Abnorm Child Psychol 36:1097-1107. https://doi.org/10.1007/s10802-008-9235-7

Riemer H, Shavitt S, Koo M, Markus HR (2014) Preferences don't have to be personal: expanding attitude theorizing with a crosscultural perspective. Psychol Rev 121:619-648. https://doi.org/ $10.1037 / \mathrm{a} 0037666$

Rohlf HL, Holl AK, Kirsch F, Krahé B, Elsner B (2018) Longitudinal links between executive function, anger, and aggression in middle childhood. Front Behav Neurosci 12:27. https://doi.org/10.3389/ fnbeh.2018.00027

Sahin-Acar B, Leichtman MD (2015) Mother-child memory conversations and self-construal in Eastern Turkey, Western Turkey and the USA. Memory 23:69-82. https://doi.org/10.1080/09658211. 2014.935437

Schmidt AF (2019) Ein kritischer Vergleich des Risk-Need-Responsivity Ansatzes und des Good Lives Modells zur Straftäterrehabilitation. Bewährungshilfe Soz Strafr Kriminalpolitik 66:211-223

Schmidt S (2018) Interkulturelle Aspekte der Kriminalprognose [Intercultural Aspects of Risk Assessment]. Dissertation. Berlin

Schmidt S, Heffernan R, Ward T (2020) Why we cannot explain crosscultural differences in risk assessment. Aggress Violent Behav 50:101346. https://doi.org/10.1016/j.avb.2019.101346

Schmidt S, van der Meer E, Tydecks S, Bliesener T (2018) How culture and migration affect risk assessment. Eur J Psychol Appl To Leg Context. https://doi.org/10.5093/ejpalc2018a7

Schmidt S, Heffernan R, Ward T The cultural agency model of criminal behavior. (submitted)

Schwartz SH (2006) A theory of cultural value orientation; explication and applications. Comp Sociol 5:137-182

Shamay-Tsoory SG, Harari H, Aharon-Peretz J, Levkovitz Y (2010) The role of the orbitofrontal cortex in affective theory of mind deficits in criminal offenders with psychopathic tendencies. Cortex 46:668-677. https://doi.org/10.1016/j.cortex.2009.04.008

Shepherd SM, Lewis-Fernandez R (2016) Forensic risk assessment and cultural diversity; Contemporary challenges and future directions. Psychol Public Policy Law 22:427-438. https://doi.org/10.1037/ law0000102

Shepherd SM, Singh JP, Fullam R (2015) Does the youth level of service/case management inventory generalize across ethnicity? Int J Forensic Ment Health 14:193-204. https://doi.org/10.1080/ 14999013.2015.1086450

Singelis TM (1994) The measurement of independent and interdependent self-construals. Pers Soc Psychol Bull 20:580-591. https:// doi.org/10.1177/0146167294205014

Singelis TM, Triandis HC, Bhawuk DPS, Gelfand MJ (1995) Horizontal and vertical dimensions of individualism and collectivism: a theoretical and measurement refinement. Cross Cult Res 29:240-275. https://doi.org/10.1177/106939719502900302

Snibbe AC, Markus HR (2005) You can't always get what you want: educational attainment, agency, and choice. J Pers Soc Psychol 88:703-720. https://doi.org/10.1037/0022-3514.88.4.703

Sterelny K (2018) Culture and the extended phenotype: Cognition and material culture in deep time. In: Newen A, Ld B, Gallagher S (Hrsg) The Oxford handbook of 4E cognition. Oxford University Press, Oxford, New York, S 773-792

Strauss-Hughes A, Heffernan R, Ward T (2019) A cultural-ecological perspective on agency and offending behaviour. Psychiatry Psychol Law 39:1-21. https://doi.org/10.1080/13218719.2019. 1644250 
Thagard P (1989) Explanatory coherence. Behav Brain Sci 12:435-467. https://doi.org/10.1017/s0140525x00057046

Thagard P (2019) Mind-society: From brains to social sciences and professions. Oxford University Press, New York

Tsai JL, Knutson B, Fung HH (2006) Cultural variation in affect valuation. J Pers Soc Psychol 90:288-307. https://doi.org/10.1037/ 0022-3514.90.2.288

Uchida Y, Kitayama S (2009) Happiness and unhappiness in east and west; Themes and variations. Emotion 9:441-456

Uchida Y, Norasakkunkit V, Kitayama S (2004) Cultural constructions of happiness: theory and emprical evidence. J Happiness Stud 5:223-239. https://doi.org/10.1007/s10902-004-8785-9

Verhoef REJ, Alsem SC, Verhulp EE, de Castro BO (2019) Hostile intent attribution and aggressive behavior in children revisited: a meta-analysis. Child Dev 90:e525-e547. https://doi.org/10.1111/ cdev. 13255

Via B, Dezember A, Taxman FS (2016) Exploring how to measure Crimenogenic needs; five instruments and no real answers. In: Taxman FS (Hrsg) Handbook on risk and need assessment. Theory and practice. Taylor and Francis, Florence, S 312-330

Vignoles VL, Owe E, Becker M et al (2016) Beyond the 'east-west' dichotomy: global variation in cultural models of selfhood. J Exp Psychol Gen 145:966-1000. https://doi.org/10.1037/xge0000175

Ward T (2016) Dynamic risk factors: scientific kinds or predictive constructs. Psychol Crime Law 22:2-16. https://doi.org/10.1080/ 1068316X.2015.1109094

Ward T (2017) Prediction and agency; The role of protective factors in correctional rehabilitation and desistance. Aggress Violent Behav 32:19-28. https://doi.org/10.1016/j.avb.2016.11.012
Ward T (2020) Why theory matters in correctional psychology. Forens Psychiatr Psychol Kriminol 14:22-34. https://doi.org/10.1007/ s11757-019-00578-9

Ward T, Carter E (2019) The classification of offending and crime related problems: a functional perspective. Psychol Crime Law 25:542-560. https://doi.org/10.1080/1068316X.2018.1557182

Ward T, Fortune C-A (2016) From dynamic risk factors to causal processes: a methodological framework. Psychol Crime Law 22:190-202. https://doi.org/10.1080/1068316X.2015.1117080

Ward T, Stewart CA (2003) The treatment of sex offenders; Risk management and good lives. Prof Psychol Res Pract 34:353-360. https://doi.org/10.1037/0735-7028.34.4.353

Ward T, Durrant R, Dixon L The classification of crime: towards pluralism. Aggression and violent behavior. (in press)

Wikstrom P-OH (2009) Individuals, settings, and acts of crime: situationalmechanisms and the explanation of crime. In: Wikström P-OH, Sampson RJ (Hrsg) The explanation of crime. Context, mechanisms and development. Cambridge University Press, Cambridge, S 61-107

Wikstrom P-OH, Treiber K (2007) The role of self-control in crime causation: beyond Gottfredson and Hirschi's general theory of crime. Eur J Criminol 4:237-264. https://doi.org/10.1177/ 1477370807074858

Wilson HA, Gutierrez L (2014) Does one size fit all?: a meta-analysis examining the predictive ability of the level of service inventory (LSI) with aboriginal offenders. Crim Justice Behav 41:196-219. https://doi.org/10.1177/0093854813500958

Wyer RS (2012) A theory of social information processing. In: van Lange P, Kruglanski A, Higgins E (Hrsg) Handbook of theories of social psychology, Bd. 1. SAGE, London, S 156-177 